\title{
Microwave-induced regeneration of activated carbons polluted with phenol. A comparison with conventional thermal regeneration
}

\author{
C. O. Ania, J.A. Menéndez, J. B. Parra*, J. J. Pis \\ Instituto Nacional del Carbón. (INCAR) C.S.I.C., Apartado 73. 33080. Oviedo, Spain.
}

\begin{abstract}
In the present work, thermal regeneration of activated carbons (AC) was carried out at $1123 \mathrm{~K}$ and under $\mathrm{N}_{2}$ atmosphere. Experiments have been carried out using a single mode microwave device operating at $2450 \mathrm{MHz}$ and a conventional electric furnace (EF) so as to compare the effect of the different heating mechanisms on the adsorptive capacities of the regenerated AC. The adsorbents were saturated with phenol in columns. Adsorptive capacities after subsequent regeneration cycles were evaluated from the breakthrough curves. Additionally, a complete textural and chemical characterization of the regenerated samples was also carried out in order to study the influence of the subsequent regeneration cycles on the texture and the adsorptive capacities of the AC. Textural characterization of the regenerated samples was carried out by means of $\mathrm{N}_{2}$ adsorption isotherms at $77 \mathrm{~K}$.
\end{abstract}

Keywords: A. activated carbon, B. heat treatment, C. adsorption, D. texture 


\section{INTRODUCTION}

Activated carbon (AC) is widely used to purify drinking water, wastewater and air. Harmful pollutants in the air and water have a high affinity to latch the porous surface of the carbonaceous adsorbent [1-3]. Treatment of industrial wastewater is also increasing in industrializing countries as industrial activity rises and the need to reuse water and reduce effluent pollution becomes greater. Formerly, when the AC reached its capacity and no longer was able to adsorb contaminates, it would simply be taken to the landfill and discarded. Discarded AC leaches into the water causing additional pollution. The increased concern of pollutants in the environment, along with more restrictive environmental regulations and increased price of activated carbon in recent years has motivated companies to develop methods to regenerate and reuse saturated activated carbon. Regeneration of AC is crucial to ensuring that the adsorption process will be economically attractive [4]. There are many methods used today to regenerate AC [5-6]. The most extensively used technique is thermal regeneration. Steam or heated nitrogen is used to destroy organic contaminates and recycle the carbon for reuse. Unfortunately, this process is time consuming and after a series of regenerations involving repetitive heating and cooling, the carbon becomes damaged. Eventually, it turns to dust and is properly disposed of. However, this process allows the activated carbon to be used again in a number of different applications.

Recently, the efficiency of applying microwave-heating technology to regenerate industrial waste activated carbon has been investigated [7-8]. The results are very promising [9-10] due to the rapid heating of the AC by microwave energy. In addition, microwave technology allowed the carbon to be recycled and reused a large number of times. This technique does not damage the carbon; rather, it increases the surface area allowing more contaminates to adhere, thereby increasing the value.

In this work, a comparative study of the regeneration of activated carbons using conventional thermal methods and a single mode microwave energy was performed. To attain this goal, regeneration of the spent AC was carried out in electric furnace (EF) and in microwave-assisted equipment (MW) under similar operation conditions. The AC were saturated with phenol columns and adsorptive capacities after successive regeneration cycles were evaluated from the breakthrough curves.

\section{EXPERIMENTAL}

A commercial AC commonly used for the decontamination of polluted waters was selected. Q is a granular activated carbon obtained from coal, which have been physically activated with steam. A particle size fraction of $0.212-0.075 \mathrm{~mm}$ was used for the studies. Prior to use, the carbon was washed several times with deionized water. Afterwards it was dried in an oven at $383 \mathrm{~K}$ for 2 days and stored in a desiccator until used.

Specific surface areas and meso- microporosity distribution were obtained from the physical adsorption of $\mathrm{N}_{2}$ at $77 \mathrm{~K}$ determined by an automatic apparatus (Micromeritics ASAP $2010 \mathrm{M}$ ). The 
surface areas and microporosity were calculated using the BET and the Dubinin-Radushkevich equations respectively [11]. On the other hand, pore volume distribution was evaluated by applying the DFT model [12].

Rapid small-scale column tests (RSSCT) were used as a feasible and less time-consuming procedure for the evaluation of the adsorptive capacity of pollutants [13]. The tests were performed at a controlled temperature of $30^{\circ} \mathrm{C}$. Breakthrough curves were obtained using $10 \mathrm{~cm}$ length stainless steel columns packed with $5 \mathrm{~g}$ of AC. All the experiments were achieved with an initial concentration of $2 \mathrm{~g} \mathrm{l}^{-1}$ of pollutant, operated at a flow rate of $4 \mathrm{ml} / \mathrm{min}$. Prior to charging the columns, all carbons were soaked in deionised water for 2 days. The outlet concentration was continuously monitored by a UV-VIS spectrophotometer at the corresponding wavelength. Adsorptive capacities were obtained by integrating over the entire breakthrough curve.

Once saturation has been reached, the exhausted AC is washed with distilled water for 2 hours, and dried overnight in an oven at $483 \mathrm{~K}$. Afterwards, the sub-samples of the exhausted AC were regenerated. Regeneration of the spent AC was carried out in an electric furnace (EF) and in a single mode microwave device (MW). The resultant samples were labeled sample-EF or sample-MW, respectively. During regeneration in the EF, the spent AC is placed in a vertical quartz reactor, while flushing a $10 \mathrm{ml} \mathrm{min}^{-1}$ flow rate of gas throughout the sample. The oxidizing or inert atmosphere is also maintained during treatment and cool-down intervals. The furnace is preheated and maintained at the temperature of the regeneration. The samples are treated at $1123 \mathrm{~K}$ and under inert $\left(\mathrm{N}_{2}\right)$ and oxidizing $\left(\mathrm{CO}_{2}\right)$ atmosphere.

The experimental equipment for the microwave regeneration of AC consists basically of a microwave magnetron of a maximum output power $2000 \mathrm{~W}$ at $2450 \mathrm{MHz}$ and a single mode cavity where the sample is exposed to microwave heating. An optical pyrometer was used to control the temperature of the sample during the regeneration process in order to prevent the sample from overheating. Exhausted activated carbon situated inside a quartz reactor is placed by means of a "chimney" into the microwave cavity and exposed to microwave heating under controlled operating conditions.

Samples will be denoted as CiRi -being $\mathrm{Ci}$ the cycles of saturation and $\mathrm{Ri}$ the number of regenerations-, followed by a reference to the atmosphere ( $\mathrm{N}$ for $\mathrm{N}_{2}$ and $\mathrm{C}$ for $\mathrm{CO}_{2}$ ) of the regeneration. For instance, sample QC3R2 NMW corresponds to $\mathrm{Q}$ activated carbon exposed to the third cycle of saturation with phenol, after two regeneration steps on the MW at under $\mathrm{N}_{2}$ atmosphere. The effect of the temperature and the atmosphere on the regeneration was also evaluated in the absence of adsorbate. For this purpose the blanks of the thermal treatment of the AC in absence of phenol were obtained. The samples will be denoted as QN and QC followed by EF or MW.

\section{RESULTS AND DISCUSSIONS}


The burn-off curve of the as-received AC was previously obtained in a thermobalance under carbon dioxide atmosphere, to determine the reaction time necessary to attain a degree of activation of $3 \%$ in the activation step. This time was also employed for the regeneration assays under $\mathrm{N}_{2}$ atmosphere. In this way, also this parameter was evaluated for the microwave furnace. The temperature reached in the carbon bed when microwave energy is applied, depends on the nature of the AC (dielectric properties) as well as on the microwave power applied to the sample [14]. For a given AC, the temperature can be modified by adjusting the input power. In the present work the established temperature of regeneration was obtained by using a variable input power, regarding the requirements of every sample. The AC reached the experimental temperature (1123 K) after 4 minutes, remaining at this temperature for the entire period of treatment. However, it was found that the input power needed to be increased with the number of the regeneration of the spent AC, ranging from 300 to 1000 Watts.

Initially, the influence of the thermal treatment on the porous structure of the selected AC in absence of adsorbate was studied. Table 1 shows the main textural parameters of the blanks treated in the EF and the MW, obtained from nitrogen adsorption data by application of BET and DR equations. It can be observed that specific surface area and micropore volume in samples treated in the MW do not differ noticeably from those in the original AC. However, changes are larger in samples treated in the EF, giving rise to samples with a lower porous structure development, compared to as-received AC. This fact is corroborated by pore volume distribution of the samples obtained by application of the DFT model at the nitrogen adsorption data (Figure 1). It was found that mesoporosity of the samples treated in the MW is nearly $20 \%$ larger than those treated in EF. At the same time, microporosity is higher in the samples treated with microwave energy (cf. Table 1). This fact occurs mostly when treatment is carried out under $\mathrm{N}_{2}$ atmosphere, whereas oxidizing atmosphere during the treatment (QC) give rise to an increase of mesopores, due to partial gasification of the activated carbon. Regarding the $\mathrm{L}$ values and the corresponding $\mathrm{E}_{\mathrm{o}}$ from DR equation, microporosity broadens with the treatment in MW. Thus, it is inferred that microwave heating of the original carbon preserves the porous structure of the carbon, compared to treatment carried out in the EF. Regarding the chemical characteristics, thermal treatment decreases the oxygen content of the AC in both devices, with a subsequent increase in the $\mathrm{ph}_{\mathrm{PZC}}$ value (cf. Table 1 ).

Heating up of the AC saturated with phenol gives rise to desorption of molecules retained on the adsorbent. The amount of gases evolved depends on the initial adsorptive capacity of the activated carbon; therefore the percentage of weight loss depends on the nature of the adsorbent, the adsorbate but also the temperature of the regeneration. It is important to point out the differences of exposition time of the carbon at high temperatures during regeneration in both devices. As it was described above, the reaction time necessary to attain a degree of activation of $3 \%$ in oxidizing atmosphere was fixed for all the regeneration assays. This parameter was evaluated for both EF (36 minutes) and MW 
(4 minutes) devices, finding a reduction of $90 \%$ when the adsorbent is treated by microwave energy. On the other hand, it was also observed that the time consumed in increase from room temperature to $1123 \mathrm{~K}$ is also lower when microwave heating is employed. The AC reached the experimental temperature after 4 minutes when heated by means of the microwave single mode device. The samples treated in the EF, however, use between 10-13 minutes to reach the operating temperature.

As a consequence of phenol desorption, changes are produced in the porous structure of the regenerated samples. It was found that BET surface area decrease with the number of regeneration cycles, being this reduction stronger when the AC is regenerated under inert (cf. Table 1) compared to oxidizing atmosphere. For instance, BET surface area of QC6R6 N is 1.6 times higher than QC6R6 C in both experimental equipments. This is due to the partial gasification on the carbon material when regeneration is carried out under $\mathrm{CO}_{2}$ atmosphere. During regeneration, phenol desorbed decomposes due to the high temperature of the treatment $(1123 \mathrm{~K})$ [15], so that sub products of phenol decomposition (carbonaceous residue) might remain inside the AC, blocking the porous structure of the adsorbent. Thus, a decrease in the surface area and a widening of the microporosity is observed. Under $\mathrm{CO}_{2}$ atmosphere, partial gasification of the carbonaceous skeleton contributes to preserve to a greater extent the porous structure. Moreover, coque deposed on the AC due to phenol decomposition might react with $\mathrm{CO}_{2}$ so that the effect of blocking the texture would be lessened.

Regeneration of saturated AC applying microwave energy seems to facilitates the desorption of the adsorbate, being this effect again more remarkable under oxidizing atmosphere (cf. Table 1). This effect might be due to the larger porous structure of the samples regenerated in the MW device. For instance, BET surface area of QC6R6 C MW is nearly 2 times higher than QC6R6 C EF (cf. Table 1). As it has been mentioned above, microwave heating of the samples preserves their porous structure, compared to treatment carried out in the EF. Microporosity is higher in the samples regenerated with microwave energy (cf. Figure 2). At the same time, it was found that mesoporosity of the samples treated in the MW is, between 20-30 \% larger than those treated in EF. This might be due to different mechanisms of heating in both devices. While heating in EF occurs by convection, microwaves heat the samples from the inside, creating a thermal gradient of temperature decreasing towards the surface of the material. This fact might be responsible for a better desorption rate of the compounds retained on the AC, giving rise to the conservation of the porous structure. However, more research has to be done in order to have a better knowledge of the results obtained, and to contrast and verify them accurately.

On the other hand, adsorptive capacities of the AC after subsequent cycles of regeneration were found to be greater by applying microwave energy, compared to the regeneration in the conventional electric furnace. Figure 3 shows a comparison of the adsorptive capacities of the original AC and the regenerated samples in both devices at the operating conditions. Regarding the effect of the atmosphere, it is inferred that that $\mathrm{CO}_{2}$ facilitates the regeneration of the exhausted AC either in 
electric or microwave heating. This fact is most likely due to the increase observed in the porous structure. However, it is important to point out the surprising differences found in later adsorptive capacities of the samples regenerated by microwave energy, compared to the EF. Using conventional heating (EF) the adsorption capacity of the AC was observed to gradually decrease with the subsequent cycles of regeneration. However, when microwave heating is applied, adsorptive capacities undergo a huge increase compared to the EF. Adsorptive capacities after the first cycle on the MW device are unexpectedly high. Afterwards, the adsorptive capacities decrease slowly. This diminution is larger when phenol is desorbed under inert atmosphere. For instance, after the first cycle -QC2R1 N-, phenol adsorption is $425 \mathrm{mg} \mathrm{g}^{-1}$ in the MW, compared to $161 \mathrm{mg} \mathrm{g}^{-1}$ in the electric furnace. After 6 cycles, the decrease in the adsorption capacity compared to the initial sample represents the $70 \%$ in the MW versus the $82 \%$ in the EF. Thus it is inferred that microwave energy contributes to increase the efficiency of regeneration of saturated AC, along with an increase of service activated carbon life.

\section{CONCLUSION}

The main advantage of using microwave energy is that the treatment can be performed in a relatively short period of time, which implies a lower consumption of gas and energy. Heating of the AC with microwave energy was found to be very rapid initially although the input power of microwaves needed to be increased when samples were saturated.

Thermal regeneration of the exhausted $\mathrm{AC}$ is favored under $\mathrm{CO}_{2}$ atmosphere, compared to inert atmosphere. This is due to partial gasification of the sample under oxidizing atmosphere -that contributes to preserve the porous structure-. Decomposition of phenol at high temperature blocks the pore structure of the adsorbent due to coque deposed inside the AC. However, this deposed products might react with $\mathrm{CO}_{2}$ so that the effect of blocking the texture would be lessened.

Microwave energy was found to preserve the porous structure of the initial samples to a great extent, as compared to samples treated in the electric furnace. Adsorptive capacities were found to be much higher when regeneration was carried out in the microwave device, even after various cycles of regeneration, compared to those obtained in the conventional furnace. Also porous structure of the samples regenerated on the MW device is larger than those regenerated in the EF, particularly their mesoporosity.

\section{ACKNOWLEDEGEMENTS}

The authors thank the European Commission, the Spanish Ministry of Science and Technology (research Project 1FD 1997-400-CO2-01) and Química Farmaceútica Bayer for financial support. 


\section{REFERENCES}

[1] Nevskaia DM, Santianes A, Muñoz V, Guerrero-Ruiz A, Carbon 1999; 37(7):1065-1074.

[2] Moreno-Castilla C, Rivera-Utrilla J, López-Ramos MV, Carrasco-Marín F, Carbon 1995; 33(7):845-851.

[3] Wang R, Chang S, J. Chem. Technol. Biotechnol 1999; 74(7):647-654.

[4] Roskill Information Services Ltd. The economics of activated carbon. Clapham Road. SW9 OJA, London, 1998:17-19.

[5] Dranca I, Lupascu T, Vogelsang K, Monahova L, J. Therm. Anal 2001; 64(3):945-953.

[6] Sheintuch M, Matatov-Meytal YI, Catal. Today 1999; 53(1):73-80.

[7] Price DW, Schmidt PS, J. Microw. Power Electromagnetic Energy 1997; 32(4):145-154.

[8] Kong Y, Cha CY, Energy Fuels 1996; 10(6):1245-1249.

[9] Komanowsky, M, J. Am. Leather Chem. Assoc 2000; 95(5):179-188.

[10] Suhm J, Am. Ceram. Soc. Bull 2000; 79(5):69-71.

[11] Dubinin MM, Progr. Surf. Membr. Sci 1975; 9:10-66.

[12] Olivier JP, Carbon 1998; 36(10):1469-1472.

[13] Cummings L, Summers RS, J. Am. Water Works Association 1994; 86(6):88-97.

[14] Bradshaw SM, van Wyk EJ, Swardt JB, J.S. Afr. Inst. Min. Metall 1998; 98(4):201-210.

[15] Cooke S, Labes MM, Carbon 1994; 32(6):1055-1058. 
Table Captions.

Table 1. Textural and chemical parameters of AC treated in EF and MW in absence and presence of adsorbate.

\begin{tabular}{lccccccc}
\hline & $\begin{array}{c}\mathrm{S}_{\mathrm{BET}} \\
\left(\mathrm{m}^{2} \mathrm{~g}^{-1}\right)\end{array}$ & $\mathrm{C}_{\mathrm{BET}}$ & $\begin{array}{c}\mathrm{W}_{\mathrm{o}} \\
\left(\mathrm{cm}^{3} \mathrm{~g}^{-1}\right)\end{array}$ & $\begin{array}{c}\mathrm{E}_{\mathrm{o}} \\
\left(\mathrm{kJ} \mathrm{mol}^{-1}\right)\end{array}$ & $\mathrm{L}(\mathrm{nm})$ & $\begin{array}{c}\text { Oxygen } \\
(\%)\end{array}$ & $\mathrm{pH}$ PZC \\
\hline Q & 1149 & 242 & 0.422 & 18.7 & 1.47 & 1.9 & 9.2 \\
\hline QN850 EF & 1022 & 353 & 0.378 & 19.7 & 1.29 & 1.1 & 9.8 \\
QC850 EF & 1064 & 308 & 0.384 & 19.6 & 1.32 & 1.2 & 10.6 \\
QN850 MW & 1083 & 309 & 0.410 & 18.8 & 1.44 & 1.4 & 10.8 \\
QC850 MW & 1088 & 285 & 0.419 & 18.8 & 1.46 & 1.6 & 11.0 \\
\hline QC1R1 ph N EF & 822 & 302 & 0.299 & 20.1 & 1.24 & 1.2 & 10.3 \\
QC6R6 ph N EF & 162 & 88 & 0.052 & 17.7 & 1.71 & 1.0 & 9.3 \\
QC1R1 ph N MW & 932 & 289 & 0.339 & 19.5 & 1.33 & 0.9 & 10.2 \\
QC6R6 ph N MW & 257 & 105 & 0.086 & 17.7 & 1.72 & 0.9 & 9.3 \\
\hline QC1R1 ph C EF & 861 & 321 & 0.313 & 20.0 & 1.26 & 1.3 & 10.6 \\
QC6R6 ph C EF & 262 & 168 & 0.086 & 17.3 & 1.64 & 1.3 & 9.3 \\
QC1R1 ph C MW & 899 & 249 & 0.317 & 20.2 & 1.23 & 0.9 & 10.0 \\
QC6R6 ph C MW & 421 & 184 & 0.147 & 19.8 & 1.29 & 1.0 & 9.2 \\
\hline
\end{tabular}


Figure Captions.

Figure 1. Pore volume distribution of the activated carbon in absence of adsorbate by application of the DFT model to $\mathrm{N}_{2}$ adsorption isotherms at $77 \mathrm{~K}$.

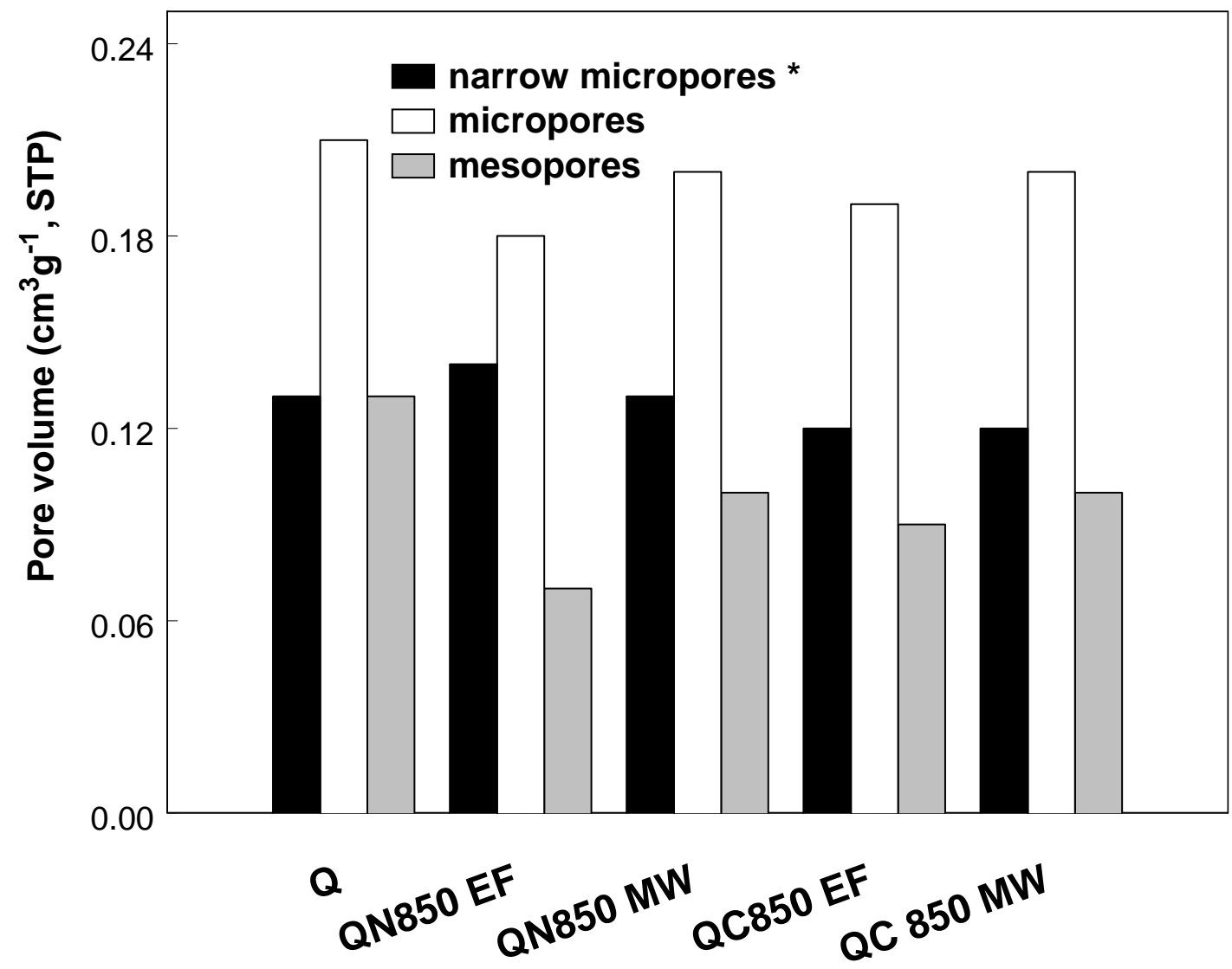

(*) narrow micropores: pore diameter dp $<0.7 \mathrm{~nm}$; micropores: $\mathrm{dp}<2 \mathrm{~nm}$ (including narrow micropores); mesopores: $2<\mathrm{dp}<50 \mathrm{~nm}$ 
Figure 2. Pore volume distribution of the regenerated activated carbons by application of the DFT model to $\mathrm{N}_{2}$ adsorption isotherms at $77 \mathrm{~K}$.

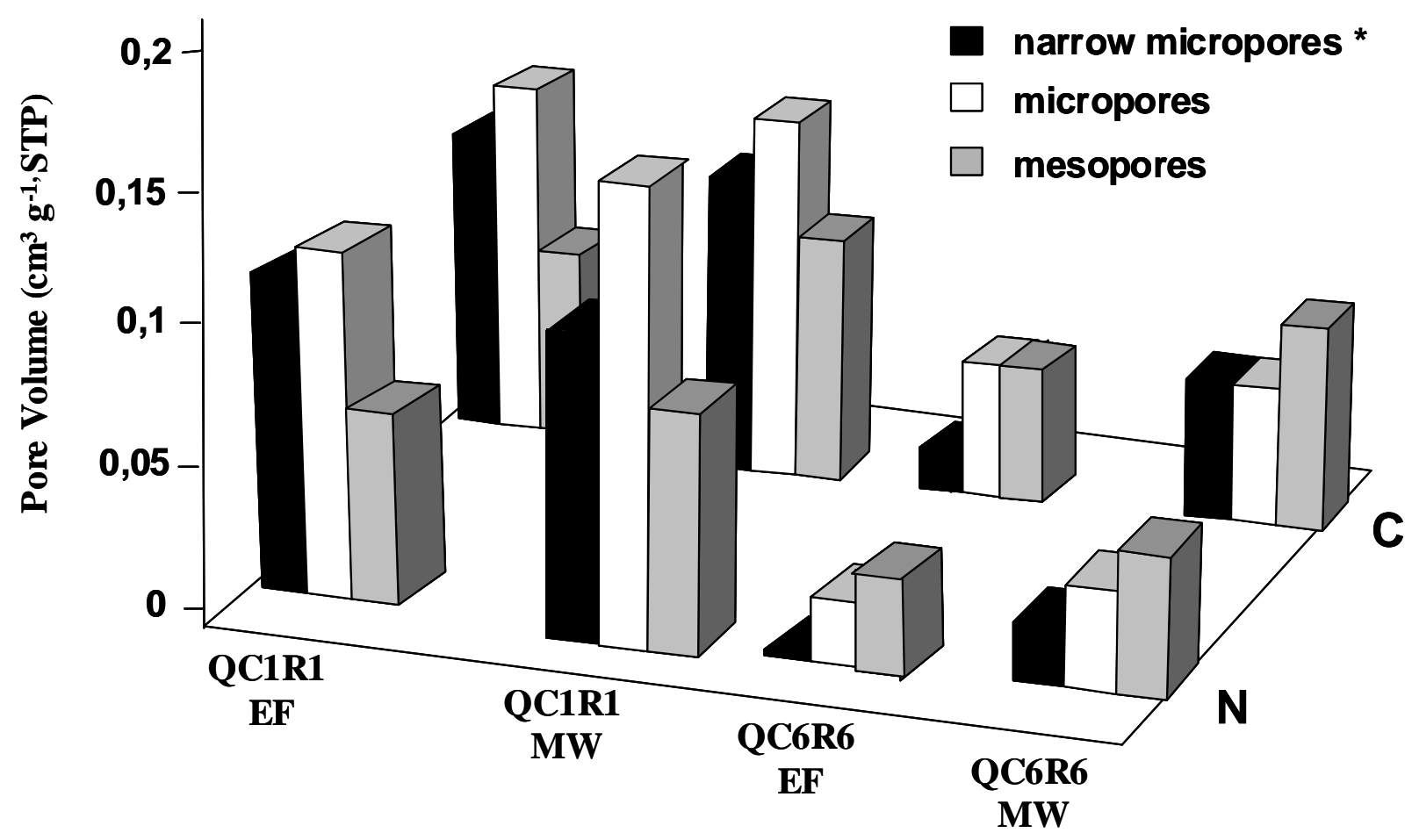

$\left(^{*}\right)$ narrow micropores: pore diameter $\mathrm{dp}<0.7 \mathrm{~nm}$; micropores: $\mathrm{dp}<2 \mathrm{~nm}$ (including narrow micropores); mesopores: $2<\mathrm{dp}<50 \mathrm{~nm}$ 
Figure 3. Adsorptive capacities of phenol after various cycles of regeneration in electric (EF) and microwave (MW) furnaces.

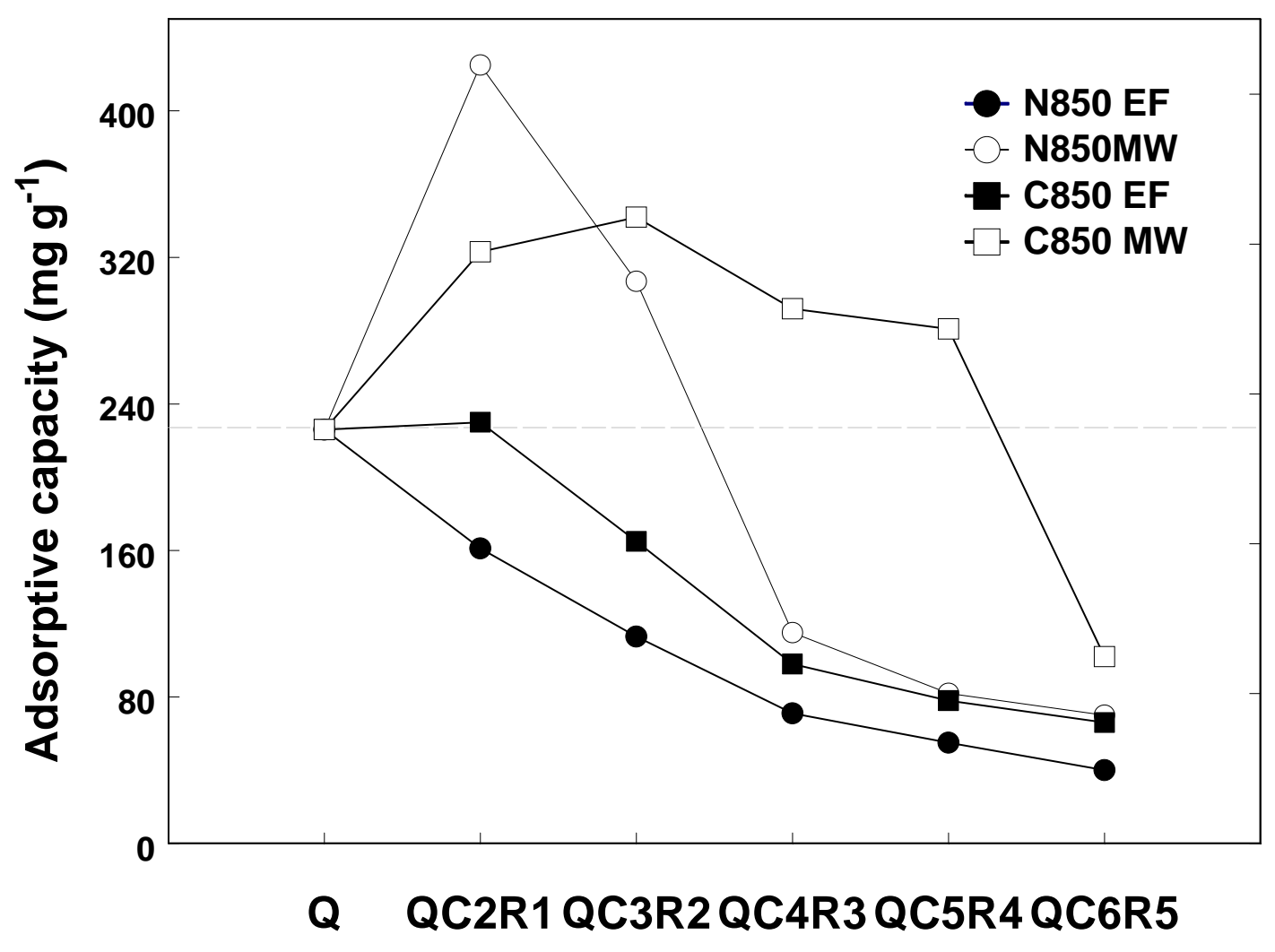

